\title{
Adiponectin as a negative regulator in obesity-related mammary carcinogenesis
}

\author{
Yu Wang ${ }^{1,2,4}$, Karen SL Lam ${ }^{3,4}$, Aimin $\mathrm{Xu}^{3,4}$ \\ ${ }^{1}$ Genome Research Center, ${ }^{2}$ Department of Biochemistry, ${ }^{3}$ Department of Medicine, and ${ }^{4}$ Research Center of Heart, Brain, Hormone \\ and Healthy Aging, University of Hong Kong, Hong Kong SAR, China
}

Cell Research (2007) 17:280-282. doi: 10.1038/cr.2007.14; published online 3 April 2007

The prevalence of obesity and its associated diseases has posed a huge healthcare impact on our society. Obesity is a major risk factor for many serious medical conditions, such as metabolic syndrome, Type 2 Diabetes and cardiovascular disorders etc. In addition, the close association of obesity with cancers has attracted significant attentions [1]. Several obesity-related cancers, including breast, prostate, endometrium, colon and gallbladder cancer, have a hormonal basis and are life style-related. Breast cancer is the most frequent cancer and the second leading cause of cancer death among women. Excess adiposity over the pre- and post-menopausal years is an independent risk factor for the development of breast cancer, and is also associated with late-stage disease and poor prognosis [2].

Adipose tissue has been shown to be an important player in obesity-related mammary carcinogenesis [2]. Adipocyte is one of the predominant stromal cell types in the microenvironment of mammary tissue. It is also the major site for local estrogen production from androgens by aromatase, thus contributing to the development of estrogen-dependent breast cancer in postmenopausal women. Additionally, the increased fat mass is associated with aberrant insulin signalling (insulin resistance) and increased insulin levels, which could directly stimulate mammary carcinogenesis. In fact, the close relationship between adipocytes and mammary tumour growth has been demonstrated by many in vitro and in vivo pharmacological studies. Mature adipocytes can promote the growth of breast carcinoma cells in a collagen gel matrix culture [3]. Co-transplantation of tumour

Correspondence: Yu Wang

Tel: 852-28199840; Fax: 852-28185653

E-mail: yuwanghk@hkucc.hku.hk cells with adipocytes into mice results in increased tumour growth and metastasis [4]. More recently, fat tissue has been recognized as an important secretory organ that can produce various hormones, cytokines and growth factors, collectively called adipokines. Dys-regulated expression and functions of these adipokines play significant roles in the pathogenesis of obesity-related diseases [5]. Interestingly, a number of adipokines, including leptin, tumour necrosis factor alpha (TNF $\alpha$ ) and interleukin 6 (IL6), etc, have been shown to promote breast cancer cell survival, proliferation and tumour development. Thus, it has been suggested that adipokines could be the key players in mediating the stromal-epithelial interactions and in influencing the growth and proliferation of tumour stroma and malignant cells in mammary gland.

Adiponectin is a circulating hormone predominantly secreted from adipocytes. Unlike many other adipokines that are up-regulated in obesity, such as TNF $\alpha$, IL-6, leptin and resistin, the circulating levels of adiponectin are inversely associated with obesity-related disorders. Many in vitro and in vivo pharmacological studies have revealed that adiponectin possesses insulin-sensitizing, anti-inflammatory, anti-diabetic and anti-atherogenic activities [6]. Replenishment of adiponectin in animal models can reduce body weight, improve glucose/lipid homeostasis, increase insulin sensitivity, prevent atherosclerosis and ameliorate fatty liver diseases. Notably, we and others have shown that adiponectin potently inhibits the proliferation of various types of cells, including aortic smooth muscle cells, myelomonocytic cells, endothelial cells and hepatic stellate cells, etc [7]. It can selectively bind to various carcinogenic growth factor and prevent the interactions of these growth factors to their respective receptors. In addition, adiponectin inhibits the growth and migration of vascular endothelial cells, prevents new blood 
vessel formation, and attenuates the growth of transplanted fibrosarcoma cell tumours in mice [8].

Several recent clinical studies have shown that obese women have reduced serum adiponectin levels and low serum adiponectin levels are significantly associated with an increased risk for breast cancer development [8]. Women with higher adiponectin levels have a reduced risk of breast cancer. Moreover, tumours in women with the low serum adiponectin levels are more likely to show a biologically aggressive phenotype. These epidemiological evidence suggests that adiponectin deficiency might be a potential mediator between obesity and breast cancer disease. Nevertheless, the detailed relationships between adiponectin expression in local mammary tissue and the development of breast cancer have not been fully established.

To test whether adiponectin could act as a negative regulator on the proliferation of human breast carcinoma cells and mammary tumour development, we have performed a series of experiments using both estrogen receptor (ER)positive and negative human breast cancer cell lines [9]. Our in vitro studies showed that adiponectin could attenuate the growth of the estrogen receptor (ER)-negative human breast carcinoma MDA-MB-231 cells by inhibiting cell proliferation and inducing apoptosis. It could also inhibit the proliferation stimulated by insulin and several other growth factors in an ER-positive human breast cancer cell line, T47D. The effects are dose-dependent and can be blocked by adiponectin specific antibodies (data not shown). These in vitro data are further corroborated by our animal study demonstrating that adiponectin supplement therapy could inhibit the breast tumour development in nude mice. In addition, we observed a much lower degree of breast tumour metastasis in mice treated with adiponectin. These results collectively suggest that replenishment of adiponectin might represent a novel therapeutic strategy for the treatment of obesity-related breast cancer.

Hyperactivation of the canonical Wnt/ $\beta$-catenin pathway is one of the most frequent signal abnormalities in many types of cancers. The central event in this pathway is the stabilization and nuclear translocation of $\beta$-catenin, where it binds to the transcription factor TCF/LEF and consequently activates a cluster of genes that ultimately establish the oncogenic phenotype. Stabilization of $\beta$-catenin protein and over-expression of cyclin D1 have been observed in over $50 \%$ of human breast tumours. Furthermore, increased $\beta$-catenin activity was found to be significantly correlated with the poor prognosis of breast cancer patients [10]. Consistent with these clinical data, numerous animal studies have demonstrated that aberrant activation of the $\mathrm{Wnt} / \beta$ catenin signaling, by either over-expression of canonical Wnt proteins or by direct stabilization of $\beta$-catenin, can lead to mammary tumorigenesis. More interestingly, Wnt signaling is also a molecular switch that governs adipogenesis. The differentiation of preadipocytes into adipocytes requires the suppression of canonical Wnt signaling, and sustained activation of $\beta$-catenin in preadipocytes blocks adipogenesis. Both mRNA and protein levels of $\beta$-catenin can be down-regulated by agonists of peroxisome proliferator-activated receptor gamma (PPAR $\gamma$ ) in 3T3-L1 adipocytes and $\mathrm{db} / \mathrm{db}$ obese/diabetic mice. Thus, further

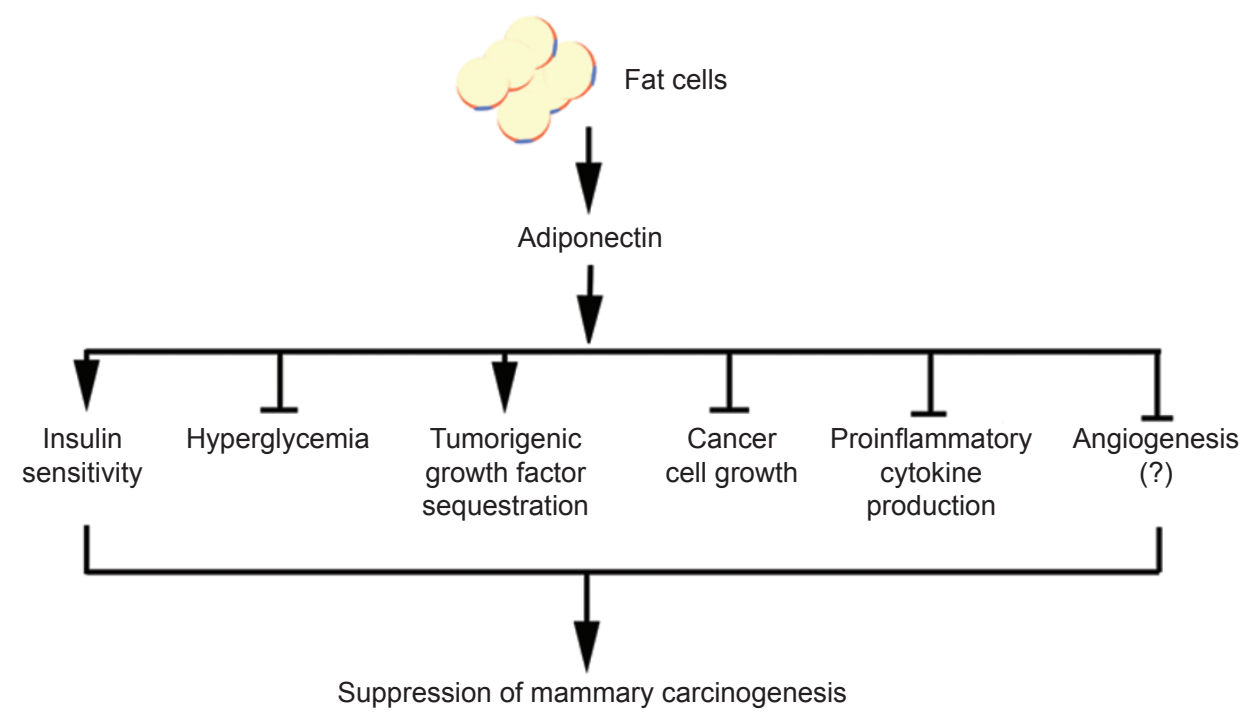

Figure 1 Adiponectin suppresses obesity-related mammary tumorigenesis via multiple mechanisms. 
understanding the cross-regulations between adipogenesis and Wnt signaling might shed new lights on obesity-related carcinogenesis.

We have demonstrated for the first time that adiponectin could modulate the GSK $3 \beta / \beta$-catenin pathway in human breast cancer cells, which might play a critical role in mediating the inhibitory effects of adiponectin on mammary tumorigenesis [9]. This conclusion is supported by our findings that chronic treatment of MDA-MB-231 cells with recombinant adiponectin markedly reduces serum-induced phosphorylation of Akt and GSK3 $\beta$, decreases intracellular accumulation and nuclear translocation of $\beta$-catenin, and suppresses cyclin D1 expression. In addition, our results have shown that chronic treatment with lithium chloride and SB-415286, two selective chemical inhibitors of GSK3 $\beta$, could attenuate the inhibitory effects of adiponectin on the expression levels of cyclin D1 and $\beta$-catenin, as well as the proliferation of MDA-MB-231 cells. Although our results showed the inhibitory effects of adiponectin on serum-induced phosphorylation of Akt at Ser473, we do not have direct evidence supporting the involvement of this kinase in adiponectin-mediated inhibition of the GSK3 $\beta / \beta$ catenin pathway in MDA-MB-231 cells. Further studies are needed to determine the role of Akt in regulating the GSK3 $\beta / \beta$-catenin pathway in our cell line and what signalling molecules at the receptor and post-receptor levels mediate adiponectin's effects. Nevertheless, our studies suggest that the cross-talks between adipokines and Wnt signaling pathways might represent a critical mechanism underlying the development of obesity-related cancers.

Given the close proximity between mammary gland cells and adipocytes, decreased adiponectin production might be causally linked to increased $\beta$-catenin accumulation and cyclin D1 over-expression observed in breast cancer patients. In addition to its direct suppressive effect on breast cancer cell proliferation, as an insulin-sensitizing hormone, adiponectin could ameliorate this disease indirectly by alleviating hyperglycemia and insulin resistance, two established risk factors for breast cancers. Furthermore, adiponectin possesses anti-inflammatory activity and can inhibit the adipocyte production of a number of inflammatory factors involved in promoting mammary tumorigenesis, such as IL6, TNF $\alpha$ and MCP-1. In addition, the anti-angiogenic activities of adiponectin could also contribute to its antitumour activities (Figure 1). Taken together, these evidence suggests that adiponectin and its agonists might represent a novel class of anticancer agents for the treatment of obesityrelated malignant tumours. Further studies are warranted to investigate the prospective association between adiponectin levels and the risk of several obesity-related cancers in humans, and to elucidate the detailed molecular events underlying the anti-tumour activities of adiponectin.

\section{References}

1 Bray GA. Medical consequences of obesity. J Clin Endocrinol Metab 2004; 89:2583-2589.

2 Lorincz AM, Sukumar S. Molecular links between obesity and breast cancer. Endocr Relat Cancer 2006; 13:279-292.

3 Manabe Y, Toda S, Miyazaki K, Sugihara H. Mature adipocytes, but not preadipocytes, promote the growth of breast carcinoma cells in collagen gel matrix culture through cancer-stromal cell interactions. J Pathol 2003; 201:221-228.

4 Elliott BE, Tam SP, Dexter D, Chen ZQ. Capacity of adipose tissue to promote growth and metastasis of a murine mammary carcinoma: effect of estrogen and progesterone. Int J Cancer 1992; 51:416-424.

5 Housa D, Housova J, Vernerova Z, Haluzik M. Adipocytokines and cancer. Physiol Res 2006; 55:233-244.

6 Matsuzawa Y, Funahashi T, Kihara S, Shimomura I. Adiponectin and metabolic syndrome. Arterioscler Thromb Vasc Biol 2004; 24:29-33.

7 Wang Y, Lam KS, Xu JY, et al. Adiponectin inhibits cell proliferation by interacting with several growth factors in an oligomerization-dependent manner. J Biol Chem 2005; 280:18341-18347.

8 Barb D, Pazaitou-Panayiotou K, Mantzoros CS. Adiponectin: a link between obesity and cancer. Expert Opin Investig Drugs 2006; 15:917-931.

9 Wang Y, Lam JB, Lam KS, et al. Adiponectin modulates the glycogen synthase kinase-3beta/beta-catenin signaling pathway and attenuates mammary tumorigenesis of MDA-MB-231 cells in nude mice. Cancer Res 2006; 66:11462-11470.

10 Brown AM. Wnt signaling in breast cancer: have we come full circle? Breast Cancer Res 2001; 3:351-355. 